\title{
pesquisa
}

BACKES FAAL; BARBOSA JG; SEDIYAMA MAN; MARTINEZ HEP; CECON PR; BARBOSA MS. 2006. Produção de lisianthus cultivado em vasos com diferentes soluções nutritivas e formas de condução. Horticultura Brasileira, 24: 6-10.

\section{Produção de lisianthus cultivado em vasos com diferentes soluções nutri- tivas e formas de condução}

\author{
Fernanda Alice AL Backes'; José Geraldo Barbosa²; Maria Aparecida N Sediyama³; Hermínia Emília P \\ Martinez $^{2}$; Paulo Roberto Cecon ${ }^{4}$; Maurício S Barbosa ${ }^{2}$ \\ ${ }^{1}$ Universidade do Contestado, Setor de Ciências Agrárias, Rua Roberto Ehlke, 86, C. Postal 01, 89460-000 Canoinhas-SC; E-mail: \\ fernanda@cni.unc.br; ${ }^{2} U F V$, Dep ${ }^{\text {to }}$. de Fitotecnia, 36571-000 Viçosa-MG; E-mail: jgeraldo@ufv.br; herminia@ufv.br; \\ mausb@bol.com.br; ${ }^{3}$ EPAMIG, Vila Gianetti, casa 46, Campus da UFV, 36571-000 Viçosa-MG; E-mail: marians@epamig.ufv.br; ${ }^{4} U F V$, \\ Dep $^{\text {to }}$. de Informática, 36571-000 Viçosa-MG; E-mail: cecon@dpi.ufv.br
}

\section{RESUMO}

O objetivo deste trabalho foi avaliar a produção da cultivar Flamenco Blue de lisianthus, em vasos fertirrigados com três soluções nutritivas e submetido a três formas de condução. O experimento foi realizado em casa-de-vegetação na UFV, de julho a outubro de 2002. O delineamento experimental foi inteiramente casualizado, com quatro repetições, fatorial $3 \times 3$. Foram avaliados a condução sem poda e poda acima do segundo e do terceiro pares de folhas e três soluções nutritivas, sendo duas provenientes da literatura e uma formulada para o lisianthus. Foram avaliados o ciclo de produção, número de hastes, número de folhas, número de botões, altura de haste, vida útil das flores, matéria fresca e seca e a concentração de nutrientes nas folhas. Para as características de crescimento não houve interação significativa entre as soluções nutritivas e as formas de condução. Não houve diferença significativa entre os resultados das soluções nutritivas, as quais são adequadas ao cultivo de lisianthus em vasos. Para as características nutricionais houve efeito de interação e efeito das soluções. A condução sem poda proporcionou ciclo mais precoce, menor número de hastes e folhas e maior altura de haste. As podas acima do segundo ou terceiro pares de folhas favoreceram a maior produção de hastes e folhas e menor altura de haste, maximizando a produção de flores.

Palavras-chave: Eustoma grandiflorum, floricultura, nutrição mineral.

\begin{abstract}
Production of lisianthus in pots with different nutrient solutions and pruning practices

The yield of lisianthus, cultivar Flamenco Blue, grown in pots with three nutrient solutions and three pruning practices was evaluated. The experiment was carried out in a greenhouse, from July to October 2002. Three pruning practices were evaluated: the absence of pruning, pruning above the second and the third pair of leaves. Two of the nutrient solutions used were taken from the literature and one was formulated specifically for the lisianthus crop. The experimental design was completely randomized with four replications and treatments arranged in a factorial design $3 \times 3$. The duration of the growing period, the number of stems, leaves and buds, height of stem, useful life of flowers, fresh and dry weight, and nutrient concentration in leaves were evaluated. No significant interactions were found among nutrient solutions and pruning practices, for growth variables. The nutrient solutions did not affect growth, but did for nutrient concentration in leaves. In the absence of pruning, the duration of growing period was shorter, the number of stems and leaves was lower and the height of the stem was higher. Pruning above the second or the third pair of leaves induced higher number of stems and leaves, smaller stem height and the higher yield of flowers.
\end{abstract}

Keywords: Eustoma grandiflorum, floriculture, mineral nutrition.

(Recebido para publicação em 22 de julho de 2004; aceito em 6 de janeiro de 2006)

$\mathrm{O}$ lisianthus (Eustoma grandiflorum Shinn.) pertencente à família Gentianaceae é uma planta nativa do Sul dos Estados Unidos, sendo encontrado principalmente nas pradarias úmidas do Nebraska e Colorado. O interesse atual da produção desta espécie ocorre principalmente pela grande diversidade de cores das flores e alta produtividade (Fox, 1998). É uma importante planta ornamental, cultivada principalmente na Europa, Japão e Estados Unidos como flor-de-corte ou planta de vaso (Halevy \& Kofranek, 1984; Ecker et al., 1994). No Brasil, foi introduzida no final da década de 80 . No entanto, o interesse econômico pela espécie começou somente a partir da década de 90 (Salvador 2000; Camargo et al., 2004).

É uma herbácea bienal, cultivada como anual, de caule ereto, com folhagem e florescimento ornamentais. O período da semeadura ao florescimento pode ser dividido em dois estádios, sendo o primeiro da germinação até o transplante, quando as plantas crescem em roseta e formam quatro pares de folhas. $\mathrm{O}$ segundo inicia pela elongação das hastes e termina com o florescimento. A principal forma de propagação é feita por sementes, as quais germinam sob temperatura de 20 a $25^{\circ} \mathrm{C}$ (Griesbach et al., 1988; Roh et al., 1989).
Segundo Frett et al. (1988) existem poucas informações quanto às necessidades nutricionais de lisianthus. No Brasil, as informações sobre a nutrição da cultura também são escassas. Camargo et al. (2004) avaliaram o crescimento e a absorção de nutrientes pelo lisianthus, cultivar Echo, produzido no solo e, observaram que as concentrações dos nutrientes na parte aérea ao final do cultivo foram, em g kg-1 de: 19,6 de N; 1,1 de P; 12,6 de $\mathrm{K} ; 0,8$ de $\mathrm{Ca} ; 1,3$ de $\mathrm{Mg} ; 2,7$ de $\mathrm{S}$; e, em mg kg-1 de: 21,9 de B; 2,9 de $\mathrm{Cu}$; 97,2 de Fe; 10,0 de Mn e 9,4 de Zn.

No cultivo de lisianthus, a poda é uma prática necessária para estimular 
brotações laterais e, conseqüentemente, a produção de maior número de hastes e de flores. A maioria das cultivares comerciais desta espécie tem haste com ramificação simpodial e as plantas não formam ramos basais, a menos que se faça uma poda acima do terceiro nó (Halevy \& Kofranek, 1984; Roh et al., 1989; Starman, 1991). Tjia \& Shechan (1986) conduziram plantas de lisianthus envasadas mantendo três a quatro pares de folhas na planta. Salvador (2000) conduziu as plantas em vaso com duas podas em épocas diferentes, sendo a primeira acima do segundo nó quando as plantas apresentavam aproximadamente $8,0 \mathrm{~cm}$ de altura e a segunda poda quando as plantas apresentavam aproximadamente $15,0 \mathrm{~cm}$ de altura.

O lisianthus, apesar de possuir grande expressão no mercado de flores, tanto para flor-de-corte quanto para vaso, é uma espécie ainda pouco pesquisada no Brasil, principalmente, quanto ao manejo da condução e da nutrição mineral. $\mathrm{O}$ trabalho teve por objetivo avaliar a produção de lisianthus cultivado em vaso com três diferentes soluções nutritivas e três formas de condução da planta.

\section{MATERIAL E MÉTODOS}

O experimento foi conduzido em casa-de-vegetação no Setor de Floricultura do Departamento de Fitotecnia da Universidade Federal de Viçosa, entre julho e outubro de 2002. A semeadura foi realizada em 03 de abril de 2002, em espuma fenólica de dimensões 5,0 x 5,0 x $3,8 \mathrm{~cm}$ previamente lavada em água corrente, colocando-se uma semente em cada célula. Após a emergência foi fornecida a solução nutritiva proposta por Barbosa et al. (2000), diluída a $12,5 \%$ da concentração inicial. Aos 30 dias a partir da semeadura, foi fornecida a solução preparada a $25 \%$ da formulação inicial.

Utilizou-se a cultivar Flamenco Blue (Sakata Seed Sudamerica), em vasos de 3 L, empregando como substrato a mistura de solo:esterco:casca de arroz carbonizada:areia $(2: 2: 1: 1)$. O transplante para os vasos foi realizado em 05 de julho de 2002, quando as mudas apresentavam entre três a quatro pares de folhas, utilizando-se três plantas por

Tabela 1. Valores médios de ciclo de produção, número de hastes por planta (NHP), número de folhas por planta (NFP), altura de haste (AH) e vida útil (VU) avaliados em lisianthus Flamenco Blue, cultivado em vaso com três formas de condução. Viçosa, UFV, 2002.

\begin{tabular}{lccccc}
\hline Podas & $\begin{array}{c}\text { Ciclo } \\
\text { (dias) }\end{array}$ & NHP & NFP & AH (cm) & VU (dias) \\
\hline Poda 11/ & $164,58 \mathrm{~b}$ & $1,00 \mathrm{~b}$ & $34,64 \mathrm{~b}$ & $61,07 \mathrm{a}$ & $22,08 \mathrm{a}$ \\
Poda 2 & $192,53 \mathrm{a}$ & $1,77 \mathrm{a}$ & $45,61 \mathrm{a}$ & $47,71 \mathrm{~b}$ & $14,58 \mathrm{~b}$ \\
Poda 3 & $187,67 \mathrm{a}$ & $1,79 \mathrm{a}$ & $45,81 \mathrm{a}$ & $43,62 \mathrm{~b}$ & $13,17 \mathrm{~b}$ \\
\hline C.V. $(\%)$ & 3,64 & 17,57 & 14,74 & 13,66 & 18,93 \\
\hline
\end{tabular}

${ }^{*}$ Médias seguidas de mesma letra na coluna não diferem entre si pelo teste Tukey a $5 \%$ de probabilidade

${ }^{1 / P o d a} 1$ - Ausência de poda; Poda 2 - Poda acima do segundo par de folhas; Poda 3 - Poda acima do terceiro par de folhas;

vaso. As fertirrigações foram realizadas diariamente, pela manhã e à tarde, aplicando-se em cada irrigação $150 \mathrm{ml}$ por vaso de solução nutritiva. Foram comparadas as soluções nutritivas de Steiner modificada (Martinez, 2002), aquela proposta por Barbosa et al. (2000) e aquela formulada para o cultivo de lisianthus. A composição dos macronutrientes $\mathrm{NO}_{3}^{-}, \mathrm{NH}_{4}^{+}, \mathrm{H}_{2} \mathrm{PO}_{4}^{-}, \mathrm{K}^{+}$, $\mathrm{Ca}^{2+}, \mathrm{Mg}^{2+} \mathrm{e} \mathrm{SO}_{4}^{2-} \mathrm{em} \mathrm{mmol} \mathrm{L}-1$, foi de 9,$0 ; 3,0 ; 1,0 ; 7,0 ; 4,5 ; 2,0$ e 3,5 na solução de Steiner modificada (Martinez, 2002); 11,51; 2,88; 1,95; 12,92; 1,51; 1,0 e 0,5 na solução Barbosa et al. (2000) e 12,$8 ; 3,2 ; 0,7 ; 6,6 ; 1,5 ; 2,7$ e 3,3 na solução formulada para lisianthus. A composição dos micronutrientes $\mathrm{B}, \mathrm{Cu}, \mathrm{Fe}, \mathrm{Mn}, \mathrm{Zn}$ e $\mathrm{Mo}$, em mmol L-1, foi de $46 ; 0,3 ; 45 ; 36 ; 1,5$ e 0,5 na solução de Steiner modificada (Martinez, 2002); de 30; 5,0; 50; 40; 2,0 e 0,1 na solução Barbosa et al. (2000) e de $30 ; 0,5 ; 60 ; 30 ; 1,5$ e 0,5 na solução formulada para o cultivo de lisianthus. As plantas foram conduzidas sem poda e com poda acima do segundo e terceiro par de folhas, realizadas 10 dias após o plantio para os vasos.

O experimento foi conduzido segundo um esquema fatorial $3 \times 3$, no delineamento inteiramente casualizado, com quatro repetições. $\mathrm{O}$ ponto de colheita foi considerado quando a primeira flor, de uma das três plantas no vaso, apresentou as sépalas totalmente abertas e as pétalas em início de abertura. Avaliou-se o ciclo de produção, considerado como o número de dias do transplante para os vasos até o ponto de colheita (Halevy \& Kofranek, 1984; Tjia \& Shechan, 1986), número de hastes por planta, número de folhas por planta, número de botões por planta, altura de haste $(\mathrm{cm})$, vida útil das flores (número de dias entre o ponto de colheita e o início da senescência da última flor aberta na haste), matéria fresca e seca total e a concentração de macro e micronutrientes nas folhas. Para as análises dos teores minerais foram coletadas todas as folhas de duas plantas do vaso, no ponto de colheita das plantas. Posteriormente, as folhas foram secas em estufa com circulação forçada de ar, a temperatura entre 70 e $75^{\circ} \mathrm{C}$, por 72 horas, pesadas e moídas e, as análises químicas dos nutrientes realizadas em extratos minerais, segundo metodologia usada no Laboratório de Nutrição Mineral de Plantas da UFV (Association of official analytical chemists, 1975; Blanchar et al., 1965; Braga \& Defelipo, 1974; Bremner, 1965; Embrapa, 1997; Jackson, 1958; Malavolta et al., 1997). Os dados foram submetidos à análise de variância, sendo as médias comparadas pelo teste de Tukey a 5\% de probabilidade.

\section{RESULTADOS E DISCUSSÃO}

Não se observou resposta das plantas às diferentes soluções, assim como interação das mesmas com as diferentes formas de condução. Observou-se menor ciclo, número de hastes e de folhas por planta quando não foi realizada a poda. Sob poda acima do segundo e do terceiro pares de folhas não foram verificadas diferenças significativas (Tabela 1). A poda é uma prática necessária para estimular brotações laterais e, conseqüentemente, a produção de maior número de hastes florais por planta. No 
Tabela 2. Concentração de nutrientes nas folhas de lisianthus Flamenco Blue cultivado em vaso sob diferentes formas de condução e soluções nutritivas. Viçosa, UFV, 2002.

\begin{tabular}{|c|c|c|c|c|c|c|c|c|c|c|}
\hline \multirow{2}{*}{ Poda } & $\mathbf{N}$ & $\mathbf{P}$ & $\mathrm{Ca}$ & $\mathbf{M g}$ & $s$ & B & $M n$ & $\mathrm{Zn}$ & $\mathrm{Cu}$ & $\mathrm{Fe}$ \\
\hline & \multicolumn{5}{|c|}{$\mathrm{g} \mathrm{kg}^{-1}$} & \multicolumn{5}{|c|}{$\mathrm{mg} \mathrm{kg}^{-1}$} \\
\hline Poda 11 & $33,7 a$ & 2,1 & $4,3 b$ & $7,7 \mathrm{~b}$ & 3,7 & 45,0 & 40,8 & $34,0 a$ & 4,7 & 178,8 \\
\hline Poda 2 & $30,7 b$ & 2,1 & $6,1 a$ & $8,5 a$ & 4,0 & 50,4 & 38,8 & $25,6 b$ & 4,7 & 219,9 \\
\hline Poda 3 & $29,9 b$ & 2,0 & $6,4 a$ & $8,4 a b$ & 3,9 & 50,1 & 37,7 & $31,4 a$ & 4,6 & 195,4 \\
\hline Solução & $\mathbf{N}$ & $\mathbf{P}$ & $\mathrm{Ca}$ & $\mathbf{M g}$ & $S$ & B & $M n$ & $\mathrm{Zn}$ & $\mathrm{Cu}$ & $\mathrm{Fe}$ \\
\hline Steiner & 31,4 & $2,0 a b$ & $6,5 a$ & $8,7 a$ & $4,2 b$ & 49,3 & 42,3 & 30,2 & 4,9 & 184,9 \\
\hline Barbosa & 31,2 & $1,9 b$ & $4,8 b$ & $7,1 \mathrm{~b}$ & $2,5 \mathrm{c}$ & 45,4 & 33,6 & 29,3 & 4,5 & 209,6 \\
\hline Lisianthus & 31,7 & $2,2 a$ & $5,5 a b$ & $8,7 a$ & $4,8 a$ & 50,7 & 41,5 & 31,7 & 4,6 & 199,5 \\
\hline$\overline{\text { C.V. }(\%)}$ & 7,75 & 9,27 & 28,33 & 8,53 & 15,51 & 19,7 & 25,70 & 17,2 & 15,9 & 24,1 \\
\hline
\end{tabular}

${ }^{*}$ Médias seguidas de mesma letra na coluna não diferem entre si pelo teste Tukey a $5 \%$ de probabilidade.

${ }^{1 /}$ Poda 1 - Ausência de poda; Poda 2 - Poda acima do segundo par de folhas; Poda 3 - Poda acima do terceiro par de folhas.

entanto, essa prática retardou o ciclo de produção. De acordo com Ferreira (1985), a eliminação do ápice da planta conduz a um estímulo ao crescimento de gemas laterais, pela redução da ação do fitohormônio auxina responsável pela inibição do crescimento das mesmas. Tjia \& Shechan (1986) cultivando lisianthus Blue em vaso, sem poda, verificaram um ciclo de produção inferior ao obtido neste estudo, em média de 114 dias após o transplante, enquanto Starman (1991), cultivando Yodel Blue, observou um ciclo de produção de 154 dias após o transplante.

Em relação à altura, observou-se que na ausência de poda as plantas apresentaram hastes mais longas, em relação àquelas que receberam poda acima do segundo ou do terceiro par de folhas, cujos valores foram 47,71 e 43,62 cm, não diferindo entre si. Harbaugh \& Scott (1996) avaliando a cultivar Flamenco Blue em diferentes épocas de semeadura obtiveram valores de altura de haste de 43, 61 e $79 \mathrm{~cm}$, com semeadura em março, junho e setembro, respectivamente. Com a cultivar Yodel Blue, em vaso, Starman (1991) observou altura de haste de $51,1 \mathrm{~cm}$.

Quanto à vida útil verificou-se que as plantas que não receberam poda apresentaram maior vida útil no vaso, em média, 22 dias, em relação àquelas podadas acima do segundo ou do terceiro par de folhas, cujas médias foram de 14,58 e 13,17 dias, não diferindo entre si. Halevy \& Kofranek (1984) observaram na cultivar Blue, também com tra- tamento de poda, uma longevidade das plantas no vaso de 18,9 dias, superior às obtidas neste trabalho.

As concentrações de fósforo, enxofre, boro, manganês, cobre e ferro não foram afetadas pela poda (Tabela 2). Observou-se que, embora na ausência de poda houve maior concentração de nitrogênio nas folhas, em todos os tipos de poda a concentração de nitrogênio está dentro da faixa proposta por Jones Jr. et al. (1991), de 25 a $35 \mathrm{~g} \mathrm{~kg}^{-1}$ de $\mathrm{N}$ na matéria seca.

As plantas que receberam poda acima do segundo ou do terceiro par de folhas apresentaram maiores concentrações de cálcio e magnésio nas folhas, porém quanto ao magnésio não houve diferença significativa entre a poda acima do terceiro par de folhas e a ausência de poda (Tabela 2). Os valores obtidos para todos os nutrientes estão dentro da faixa proposta por Jones Jr. et al. (1991), que é de 1,5 a $10 \mathrm{~g} \mathrm{~kg}^{-1}$ de P; 2 e $3 \mathrm{~g} \mathrm{~kg}^{-1}$ de $\mathrm{Ca} ; 1,5$ a $10 \mathrm{~g} \mathrm{~kg}^{-1}$ de $\mathrm{Mg}$ e 1,5 a $5 \mathrm{~g} \mathrm{~kg}^{-1}$ de $\mathrm{S}$ na matéria seca em tecidos vegetais. Camargo et al. (2004) observaram concentrações de macronutrientes inferiores às obtidas neste experimento na parte aérea de lisianthus cultivar Echo, cultivado no solo.

Quanto aos micronutrientes, foi observado efeito significativo entre as podas apenas para zinco. Na ausência de poda e na poda acima do terceiro par de folhas, foram observados valores superiores de concentração de zinco nas folhas de lisianthus, em média 34,0 e $31,4 \mathrm{mg} \mathrm{kg}^{-1}$ de $\mathrm{Zn}$, quando compara- dos à poda acima do segundo par de folhas, com concentração média de 25,6 $\mathrm{mg} \mathrm{kg}^{-1}$ de $\mathrm{Zn}$ (Tabela 2). Esses valores estão dentro da faixa proposta por Jones Jr. et al. (1991) e Marschner (1995) que sugerem valores entre 15 e $50 \mathrm{mg} \mathrm{kg}^{-1}$ de Zn na matéria seca de tecidos vegetais. Harbaugh \& Woltz (1991) encontraram nas folhas de lisianthus cultivar Saga Purple valores de $32 \mathrm{mg} \mathrm{kg}^{-1}$ de Zn, portanto uma concentração próxima à obtida neste trabalho. Camargo et al. (2004) verificaram concentração inferior de 9,4 $\mathrm{mg} \mathrm{kg}^{-1}$ de $\mathrm{Zn}$ na parte aérea da cultivar Echo.

Todos os valores encontrados para os demais micronutrientes estão dentro da faixa proposta por Jones Jr. et al. (1991) de 20 a $70 \mathrm{mg} \mathrm{kg}^{-1}$ de B, 10 a 50 $\mathrm{mg} \mathrm{kg}^{-1}$ de $\mathrm{Mn}, 3$ a $7 \mathrm{mg} \mathrm{kg}^{-1}$ de Cu e 10 a $1000 \mathrm{mg} \mathrm{kg}^{-1}$ de $\mathrm{Fe}$, e próximas às obtidas por Harbaugh \& Woltz (1991) de $42 \mathrm{mg} \mathrm{kg}^{-1}$ de $\mathrm{Mn}$ e $3 \mathrm{mg} \mathrm{kg}^{-1}$ de $\mathrm{Cu}$, enquanto Camargo et al. (2004) verificaram, para os micronutrientes analisados na parte aérea da cultivar Echo, valores inferiores aos obtidos neste experimento.

As concentrações de fósforo, cálcio, magnésio e enxofre nas folhas de lisianthus foram influenciadas pelas soluções nutritivas (Tabela 2), enquanto que, para nitrogênio não foi detectado efeito significativo. Para o fósforo, apesar da solução formulada para o cultivo de lisianthus apresentar menor concentração na sua composição química $(0,7$ mmol P L $\left.{ }^{-1}\right)$, observou-se maior concentração do elemento nas folhas de plan- 
tas cultivadas nesta solução em relação à solução proposta por Barbosa et al. (2000). Esses valores estão próximos ao limite inferior proposto por Jones Jr. et al. (1991) de 1,5 a $10 \mathrm{~g} \mathrm{~kg}^{-1}$ de $\mathrm{P}$ na matéria seca. Harbaugh \& Woltz (1991) encontraram valores inferiores em plantas de lisianthus cultivar Saga Purple, de $1,2 \mathrm{~g} \mathrm{~kg}^{-1}$ de $\mathrm{P}$, quando comparados aos obtidos neste trabalho com Flamenco Blue.

Verificou-se que as soluções nutritivas de Steiner modificada e aquela formulada para o cultivo de lisianthus proporcionaram maior acúmulo de cálcio e magnésio nas folhas, embora em relação à concentração de cálcio a solução formulada para lisianthus não diferiu da solução proposta por Barbosa et al. (2000). Provavelmente, essas concentrações estão relacionadas à composição química inicial das soluções, de 4,5 mmol Ca L ${ }^{-1}$ e 2,0 mmol $\mathrm{Mg} \mathrm{L}^{-1}$ na solução de Steiner modificada, e de 2,7 mmol Mg L-1 na solução formulada para lisianthus. Os valores de concentração obtidos, independente da solução nutritiva utilizada, estão acima do valor encontrado por Harbaugh \& Woltz (1991) com a cultivar Saga Purple, de 3,9 $\mathrm{g} \mathrm{kg}^{-1}$ de $\mathrm{Ca}$ e de 4,3 $\mathrm{g} \mathrm{kg}^{-1}$ de $\mathrm{Mg}$.

Em relação ao enxofre, a solução formulada para lisianthus favoreceu maior acúmulo do elemento nas folhas, em média, 4,8 $\mathrm{g} \mathrm{kg}^{-1}$, enquanto que a solução de Steiner modificada apresentou concentração média de $4,2 \mathrm{~g} \mathrm{~kg}^{-1}$. A menor concentração de enxofre nas folhas de 2,5 $\mathrm{g} \mathrm{kg}^{-1}$ foi verificada com a utilização da solução proposta por Barbosa et al. (2000). Tal comportamento deve-se ao fato de a solução formulada para lisianthus e a Steiner modificada apresentarem na sua composição química teores superiores à solução proposta por Barbosa et al. (2000), de 3,5, 3,3 e $0,5 \mathrm{mmol} \mathrm{S} \mathrm{L}^{-1}$, respectivamente. As concentrações de enxofre obtidas nas folhas de Flamenco Blue estão dentro do intervalo de 1,5 e $5 \mathrm{~g} \mathrm{~kg}^{-1}$ proposto por Jones Jr. et al. (1991) e Marschner (1995) na matéria seca de tecidos vegetais.

Não foram detectados efeitos significativos entre as soluções nutritivas quanto aos micronutrientes analisados nas folhas de lisianthus cultivar Flamenco Blue (Tabela 2). Entretanto,

Tabela 3. Valores médios de concentração de $\mathrm{K}$, em $\mathrm{g} \mathrm{kg}^{-1}$, nas folhas de lisianthus para as combinações de soluções e formas de condução. UFV, Viçosa, 2002.

\begin{tabular}{lccc}
\hline Poda & Steiner & Barbosa & Lisianthus \\
\hline Poda 1 & 54,5 A a & $48,7 \mathrm{~A} \mathrm{ab}$ & $44,5 \mathrm{~A} \mathrm{~b}$ \\
Poda 2 & $55,5 \mathrm{~A} \mathrm{a}$ & $55,3 \mathrm{~A} \mathrm{a}$ & $44,5 \mathrm{~A} \mathrm{~b}$ \\
Poda 3 & $42,0 \mathrm{~B} \mathrm{a}$ & $48,7 \mathrm{~A} \mathrm{a}$ & $42,2 \mathrm{~A} \mathrm{a}$ \\
\hline C.V. (\%) & & 8,24 & \\
\hline
\end{tabular}

${ }^{*}$ Médias seguidas de mesma letra maiúscula na coluna e minúscula na linha não diferem entre si pelo teste Tukey a 5\% de probabilidade.

${ }^{1 /}$ Poda 1 - Ausência de poda; Poda 2 - Poda acima do segundo par de folhas; Poda 3 - Poda acima do terceiro par de folhas.

esses valores estão dentro dos citados por Jones Jr. et al. (1991).

A análise de variância indicou não existir interações significativas entre soluções nutritivas e diferentes formas de condução quanto às características observadas, exceto para a concentração de potássio nas folhas (Tabela 3). Observa-se que a menor concentração de potássio foi obtida utilizando-se a poda acima do terceiro par de folhas em Flamenco Blue cultivado na solução de Steiner modificada. Concentrações superiores de potássio com a mesma solução nutritiva foram obtidas na ausência de poda e na poda acima do segundo par de folhas (Tabela 3). Essas concentrações encontram-se próximas ao intervalo superior de concentração nas folhas proposto por Jones Jr. et al. (1991), que é de 10 a $50 \mathrm{~g} \mathrm{~kg}^{-1}$ de $\mathrm{K}$ na matéria seca. A solução recomendada por Barbosa et al. (2000) e aquela formulada para lisianthus apresentaram resultados similares, independente do tipo de poda realizado nas plantas. No entanto, todos os valores de concentração obtidos condizem com os propostos por Marschner (1995), de 25 a $50 \mathrm{~g} \mathrm{~kg}^{-1}$ de K na matéria seca.

Em relação às concentrações obtidas em plantas que não receberam poda, observou-se que a maior concentração foi obtida com a utilização da solução de Steiner modificada a qual não diferiu significativamente de plantas cultivadas com a solução proposta por Barbosa et al. (2000). Na utilização da poda acima do segundo par de folhas, as sconcentrações de potássio foram verificadas nas soluções de Steiner modificada e Barbosa et al. (2000), com médias de 55,5 e 55,3 $\mathrm{g} \mathrm{kg}^{-1}$ de $\mathrm{K}$, respectivamente. Na poda acima do tercei- ro par de folhas, independente da solução nutritiva utilizada, observou-se que não houve diferença significativa entre as concentrações de potássio nas folhas. Harbaugh \& Woltz (1991) encontraram nas folhas da cultivar Saga Purple concentração de $36 \mathrm{~g} \mathrm{~kg}^{-1}$ de $\mathrm{K}$, valor inferior aos obtidos neste trabalho.

Conclui-se que a produção de lisianthus é maximizada com a poda acima do segundo ou do terceiro pares de folhas, resultando em maior número de hastes e folhas e menor altura de hastes. As soluções nutritivas avaliadas são adequadas para o cultivo do lisianthus em vaso.

\section{LITERATURA CITADA}

ASSOCIATION OF OFFICIAL ANALYTICAL CHEMISTS, AOAC. 1975. Official methods of analysis. $12 \mathrm{ed}$. Washington, D.C. $1094 \mathrm{p}$.

BARBOSA JG; KAMPF AN; MARTINEZ HEP; KOLLER OC; BOHNEN H. 2000. Chrysanthemum cultivation in expanded clay. I. Effect of the nitrogen-phosphorus-potassium ratio in the nutrient solution. Journal of Plant Nutrition 23: 1327-1336.

BLANCHAR RW; REHM G; CALDWELL AC. 1965. Sulfur in plant material by digestion with nitric and perchloric acid. Proceedings Soil Science Society of America 29: 71-72.

BRAGA JM; DEFELIPO EV. 1974. Determinação espectrofotométrica de fósforo em estratos de solos e plantas. Revista Ceres 21: 73-85.

BREMNER JM. 1965. Total nitrogen. In: BLACK CA. (Ed.). Methods of soil analysis. Madison: American Society of agronomy. p. 1149-1178.

CAMARGO MS; SHIMIZU LK; SAITO MA; KAMEOKA CH; MELLO SC; CARMELLO QAC. 2004. Crescimento e absorção de nutrientes pelo lisianthus (Eustoma grandiflorum) cultivado em solo. Horticultura Brasileira 22: 143146.

ECKER R; BARZILAY A; OSHERENKO E. 1994. The genetic relations between length of time to germination and seed dormancy in lisianthus (Eustoma grandiflorum). Euphytica 80: 125-128. 
EMPRESA BRASILEIRA DE PESQUISA AGROPECUÁRIA - EMBRAPA. 1997. Manual de métodos de análise de solo. 2. ed. Rio de Janeiro: Embrapa Solos. 212 p.

FERREIRA JFS. 1985. Efeito de podas para a produção de ramos porta-borbulhas do surto primaveril dos citros. Lavras: ESAL. 80 f. (Dissertação de Mestrado)

FOX R. 1998. Lisianthus - a specialty cut flower Practical Hydroponics \& Greenhouses: 43-51.

FRETT JJ; KELLY JW; HARBAUGH BK.; ROH M. 1988. Optimizing nitrogen and calcium nutrition of lisianthus. Communications in Soil Science Plant Analysis 19: 13-24.

GRIESBACH RJ; SEMENIUK P; ROH M; LAWSON RH. 1988. Tissue culture in the improvement of Eustoma. HortScience 23: 790791.
HALEVY AH; KOFRANEK AM. 1984. Evaluation of lisianthus as a new flower crop. HortScience 19: 845-847.

HARBAUGH BK; WOLTZ SS. 1991. Eustoma quality is adversely affected by low $\mathrm{pH}$ of root medium. HortScience 26: 1279-1280.

HARBAUGH BK; SCOTT JW. 1996. 'Maurine Blue' [Eustoma grandiflorum (Raf.) Shinn.]. HortScience 31: 1055-1056.

JACKSON ML. 1958. Soil chemical analysis. Englewood Cliffs: Prentice Hall. 458p.

JONES JR JB; WOLF B; MILLS HA. 1991. Plant Analysis Handbook - a practical sampling, preparation, analysis, and interpretation guide. Geórgia: Micro-Macro Publishing. 213 p.

MALAVOLTA E; VITTI GC; OLIVEIRA SA. 1997. Avaliação do estado nutricional das plantas: princípios e aplicações. 2. ed. Piracicaba: Potafos. 319 p.
MARSCHNER H. 1995. Mineral nutrition of higher plants. Londres: Academic Press, 889 p. MARTINEZ HEP. 2002. O uso do cultivo hidropônico de plantas em pesquisa. Viçosa: UFV. $61 \mathrm{p}$ (Caderno Didático 1).

ROH MS; HALEVY AH; WILKINS HF. 1989. Eustoma grandiflorum. In: HALEVY AH. (Ed.) Handbook of Flowering. Florida: CRC Press. p. 322-327.

SALVADOR ED. 2000. Caracterização física e formulação de substratos para o cultivo de algumas ornamentais. Piracicaba: ESALQ. 148 f. (Dissertação de Doutorado).

STARMAN TW. 1991. Lisianthus growth and flowering responses to uniconazole. HortScience 26: $150-152$.

TJIA B; SHECHAN TJ. 1986. Chemical height control of Lisianthus russellianus. HortScience 21: 147-148. 